\section{An outbreak of epidemic keratoconjunctivitis at an outpatient ophthalmology clinic}

\author{
Timothy J. Doyle, ,'2 Diane King, \\ Judith Cobb, ' Darlene Miller, ${ }^{3}$ \\ Barbara Johnson, ${ }^{1}$
}

${ }^{1}$ Florida Department of Health;'² Centers for Disease Control and Prevention, Career Epidemiology Field Officer Program; ${ }^{3}$ Bascom Palmer Eye Institute; University of Miami, Miami, FL, USA

\section{Abstract}

Epidemic keratoconjunctivitis (EKC) is an acute eye infection caused by adenovirus. We investigated an outbreak of EKC at an outpatient ophthalmology practice in the context of a suspected community wide increase in EKC activity. A site visit was made to the facility reporting the outbreak. A line list was created of patients clinically diagnosed with EKC at the practice during the previous 5 months. A questionnaire was faxed to all other licensed ophthalmologists in the county regarding recent EKC activity in their facility. Descriptive data analyses were conducted. The outbreak facility reported 37 patients clinically diagnosed with EKC during the previous 5 months. In addition, the single ophthalmologist at the practice also had symptoms compatible with EKC during the outbreak period. Specimens were collected on 4 patients and all were positive for adenovirus serotype 8 . Forty percent of ophthalmologists surveyed in the county saw at least one EKC patient in the previous 3 months, and 20\% reported a perceived increase in EKC activity in recent months over normal seasonal patterns. The outbreak at the facility likely began as part of a widespread community increase in EKC that may have been amplified at the facility through nosocomial transmission. Medical providers experiencing increases in EKC activity above seasonally expected norms should contact their public health department for assistance with etiologic diagnoses and outbreak control.

\section{Introduction}

Epidemic keratoconjunctivitis (EKC) is an acute infection of the eye caused by adenovirus, of which several different types have been implicated. Common manifestations of this infection include inflammation of the conjunctivae, edema of the eyelid, pain, photophobia, and blurred vision. ${ }^{1}$ Formation of a mem- brane or pseudomembrane may also occur. Complications include conjunctival scarring and symblepharon. No specific treatment exists. Transmission may occur through direct contact with eye secretions of an infected person, or indirectly through contact with contaminated surfaces, instruments or solutions. EKC is highly contagious and frequently occurs in epidemic fashion, as its name implies. The incubation period can range from 2 days to 2 weeks, with an average of 8-10 days, and infected people remain contagious for up to 2 weeks..$^{24}$ Viral particles can remain infectious on surfaces for up to a month. ${ }^{2}$

In March 2009, the Florida Department of Health (FLDOH) was contacted by a regional academic ophthalmology referral center, regarding a local outpatient ophthalmology practice (practice A) that had requested assistance with an outbreak of EKC at the practice. Practice A was contacted and a site visit was arranged. At the same time, anecdotal reports were noted of increased EKC activity throughout the county. To assess whether the problems experienced at practice A were isolated or part of a more wide-spread increase in EKC activity, a brief survey was developed of outpatient ophthalmology clinics in the county. This report describes the investigation of the EKC outbreak at practice A and the survey results of other out-patient ophthalmology clinics in the county.

\section{Materials and Methods}

\section{Investigation of practice $A$}

Health department staff visited practice A during service hours to observe patient care procedures. The practice consists of a main office (office A1) and an auxiliary office (office A2), located in different parts of the county, and only office Al was visited by investigators. Office staff assembled a line list from patient charts of all persons diagnosed with EKC at practice A during the previous 5 months. Confirmed cases were defined as those with compatible clinical symptoms of EKC and laboratory confirmed adenovirus infection through viral culture of ocular swab specimens. Suspect cases were defined as those with a clinical diagnosis of EKC, without laboratory confirmation. The line list included the onset date of symptoms, dates previously seen in the practice prior to symptoms, and which office visited. Based on the estimated outside incubation period for EKC of 2 weeks, cases were further classified as possibly healthcare associated if they were seen at practice A for an unrelated condition within 2weeks of the onset of EKC symptoms. Specific information regarding medical procedures performed during previous visits or past visits to
Correspondence: Florida Department of Health, 4052 Bald Cypress Way, Bin A-12, Tallahassee, FL 32399-1720 USA. E-mail: tdoyle@cdc.gov

Key words: epidemic keratoconjunctivitis, outbreak, adenovirus.

Acknowledgements: the authors would like to thank Drs. Dean Erdman and Eileen Schneider for assistance with laboratory testing and the physician and staff of practice A for their extensive cooperation and assistance during the investigation.

Conflict of interest: the authors report no conflicts of interest.

Contributions: TD, DK and BJ conducted the outbreak investigation; JC conducted the survey of providers in the county. DM conducted the laboratory testing; TD prepared the tables and figures. TD, DK, and DM prepared the manuscript; JC and BJ provided critical review of the draft manuscript.

Received for publication: 3 September 2010. Accepted for publication: 11 November 2010.

This work is licensed under a Creative Commons Attribution 3.0 License (by-nc 3.0).

(C) Copyright T.J. Doyle et al., 2010

Licensee PAGEPress, Italy

Infectious Disease Reports 2010; 2:e17

doi:10.4081/idr.2010.e17

other providers was not obtained. During the site visit, a rapid test was performed on 2 patients with compatible symptoms, using the Adeno Detector ${ }^{\mathrm{TM}}$ test kit [www.rpttests.com/products_ad.html]. Specimens for viral culture were obtained from the same 2 patients and from 2 additional patients seen the next day. Viral cultures were performed at the Bascom Palmer Eye Institute, microbiology laboratory, in Miami, FL. Viral culture was conducted by inoculation of A549 and MRC cells (Viromed Laboratories, Minnetonka, MN, USA) and incubation for up to 21 days, followed by immunoflourescent staining with monoclonal antibody (PathoDX, Remel, Lenexa, KS, USA). Viral culture isolates positive for adenovirus were forwarded to the Centers for Disease Control and Prevention (CDC) for viral serotyping by polymerase chain reaction, using primers specific for adenovirus serotypes.

\section{Survey of outpatient ophthalmology clinics}

FLDOH developed a list of all licensed ophthalmologists in the county from medical licensing records. A cover letter and brief 1page questionnaire were faxed to all ophthalmology practices in the county alerting them to 
the possible EKC problem, and requesting the completion and return of the questionnaire. The questionnaire included items regarding their perceptions of recent EKC incidence, number of EKC cases diagnosed in their clinic during the last three months, similar illness among office staff, and any laboratory test results available. Analyses of data collected from both practice A and the county wide survey were descriptive and no statistical tests of significance were performed.

\section{Results}

\section{Investigation of practice $A$}

Practice A is a busy, outpatient ophthalmology practice that serves a predominantly elderly patient population and sees approximately 150-200 patients per week. The practice has 1 general ophthalmologist (physician A), specializing in cataract and corneal surgery, who founded the practice and has worked for several years in the community. At office A1, there are 4 exam rooms and 2 ophthalmology technicians who assist with patient care; 2 other staff assist with administrative duties. Office A2 is a smaller satellite facility where physician A and the technicians work 2 half-days per week. Approximately two-thirds of patient visits occur at office $\mathrm{Al}$, where all patient records are maintained for the practice.

Interviews with staff revealed that the first EKC patient believed to be part of this outbreak was seen in office A1 in late November 2008. A total of 37 patients were clinically diagnosed with EKC at practice A from November 2008 through March 2009. Twenty-three (62\%) EKC patients were seen at office A1, $12(32 \%)$ at office A2, and 2 patients were seen at both offices (Figure 1). Of the 4 patients seen at office A1 for whom specimens were collected for viral culture, all 4 were positive for adenovirus, serotype 8 . Two of the four laboratory confirmed patients had rapid tests performed during the clinical visit, and both of these patients were negative for adenovirus by rapid test. All of the laboratory confirmed patients had a previous visit at office Al within the previous 2 weeks, prior to their symptom onset.

Interviews revealed that physician A had symptoms compatible with EKC, with onset of these symptoms on approximately February 5 , 2009 and lasting approximately 4-5 weeks before resolution of symptoms. No other staff members had similar symptoms and no diagnostic specimens were collected from any staff members. Among the other 37 confirmed or suspected EKC patients, the median age was 79 years (Table 1). Nineteen EKC patients (51\%) had been seen for an unrelated condition by physician A, within 2 weeks of the onset of their symptoms (Figure 2). Four addi-

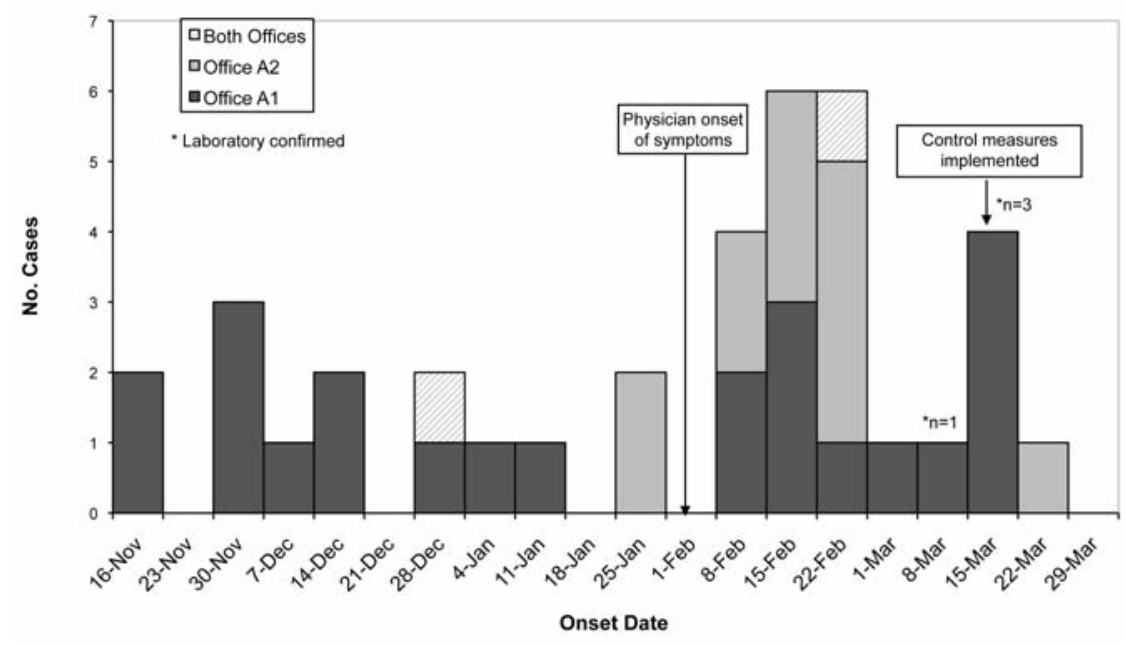

Figure 1. Epidemiologic curve of epidemic keratoconjunctivitis patients seen at practice A by office location.

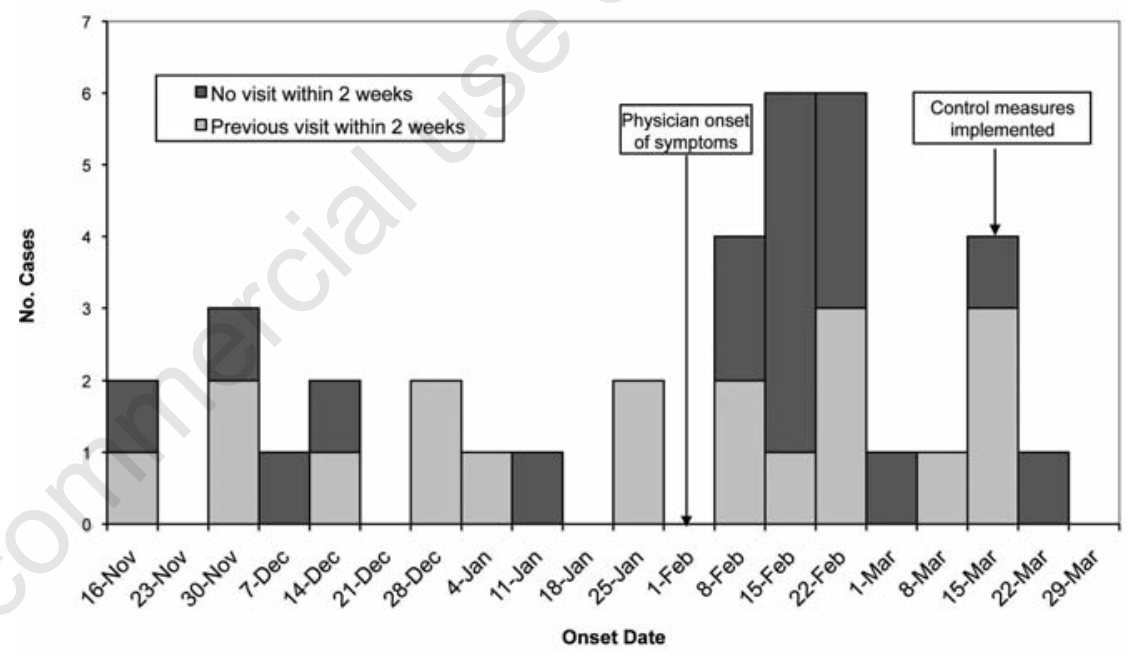

Figure 2. Epidemiologic curve of epidemic keratoconjunctivitis patients seen at practice A by history of recent office visit.

tional EKC cases were seen at practice A between 15 and 17 days prior to symptom onset. Of the 19 EKC patients seen at practice A within 2 weeks of symptom onset, 13 had been seen at office A1, 5 at office A2, and 1 at both offices.

On March 18, 2009, the day the health department was notified of the outbreak, practice A was closed for terminal cleaning. All surfaces were wiped by office staff with a bleach solution, reusable vials of drops were discarded, and tonometers and other medical equipment were thoroughly cleaned. When practice A opened the following day, one exam room in office A1 was dedicated exclusively for EKC patients, and EKC and non-EKC patients were separated on different sides of the waiting
Table 1. Demographic and clinical characteristics of Epidemic keratoconjunctivitis patients seen at practice $A$.

\begin{tabular}{lc}
$\begin{array}{l}\text { Sex, female } \\
\text { Median age (range) }\end{array}$ & $79(15-99)$ \\
\hline $\begin{array}{l}\text { Office visit within 2 weeks } \\
\text { before onset }\end{array}$ & $19 / 37(51 \%)$ \\
Bilateral symptoms & $30 / 37(81 \%)$ \\
\hline Redness & $28 / 37(76 \%)$ \\
Pain & $16 / 37(43 \%)$ \\
\hline Tearing & $14 / 37(38 \%)$ \\
Irritation & $4 / 37(11 \%)$ \\
\hline Decreased visual acuity & $1 / 37(3 \%)$ \\
Laboratory confirmed & $4 / 37(11 \%)$ \\
\hline
\end{tabular}


room. For patient encounters, office staff implemented aggressive use of gloves and wiping of surfaces with germicidal wipes following each patient encounter. These control measures were initiated independently by practice A, prior to the health department becoming involved in the investigation. Following implementation of these control measures, 2 additional cases were diagnosed with EKC at practice A, 2 and 6 days respectively following the terminal cleaning. The patient with onset 2 days after implementation of control measures had a previous office visit 15 days earlier; the other patient had not been seen in practice A during the past year. Since prevention measures had already been implemented and the outbreak had begun to dissipate by the time the epidemiologic investigation was under way, no environmental samples were collected and extensive analyses were not conducted to identify additional risk factors associated with infection.

No additional EKC patients were seen at practice $\mathrm{A}$ in the two months following this outbreak, however, physician A reported seeing 4 additional EKC patients within 6 months following the outbreak. Approximately 8 EKC case patients from the outbreak, including physician A, developed post-viral keratitis, requiring long-term topical steroid treatment to control corneal infiltrates.

\section{Survey of outpatient clinics in the community}

Medical licensing records revealed 29 ophthalmologists in general practice in the county, including physician A. In April 2009, questionnaires were faxed to all 28 providers, other than physician $\mathrm{A}$, and responses were received from 20 (71\%). Of those who responded, 4 (20\%) reported an increase in conjunctivitis during the previous 6 months above normal seasonal patterns. Eight of twenty (40\%) respondents believed they had seen patients from January through March 2009 with symptoms compatible with EKC or acute hemorrhagic conjunctivitis (AHC). Among these providers, the median number of EKC/AHC patients seen per clinic during this period was 10 (range 1 to 25) with a total of 84 patients with compatible symptoms seen at these 8 clinics. None of these 84 suspect case-patients were laboratory confirmed. For comparison, during the same 3-month period, physician A saw 28 EKC patients, making 112 total EKC patients for all 21 providers. Because denominator data were not collected on the total number of patients seen at each clinic, attack rates at each clinic cannot be calculated.

Two of the eight clinics (25\%) reported seeing symptoms compatible with EKC in patients who had visited their clinic in the previous 2 weeks for an unrelated problem. Of these 2 clinics, one reported seeing EKC symptoms in 10 return patients, and the other clinic reported $1 \mathrm{EKC}$ case in a return patient. None of the 20 clinics who responded to the survey reported similar symptoms among clinic staff. Finally, 7 of 18 (39\%) clinics that responded indicated future interest in submitting specimens to reference laboratories for diagnostic testing.

\section{Discussion}

The outbreak at practice A described in this report involved 37 patients diagnosed with EKC over a 5 month period. Approximately one-half of case-patients had recently been seen by physician A for an unrelated condition, and previous visits occurred at both offices of the practice. One additional suspect case involved the ophthalmologist, raising the strong possibility of iatrogenic transmission within the practice. At least 2 other ophthalmology clinics in the county reported a similar number of case-patients during the same time period and at least 1 of these clinics may also have had nosocomial transmission, based on the large number of EKC patients who were recently seen for unrelated conditions. Patients diagnosed with EKC were also seen in several other clinics throughout the county during this time frame. Taken together, these observations suggest broad distribution of EKC-like illness in the community with focal amplification in practice A, likely due to nosocomial transmission.

The etiologic agent identified in 4 EKC patients at practice A was adenovirus, serotype 8 (Ad8). Ad8 is a common cause of severe EKC, along with adenoviral serotypes 19 and 37.,6 Other serotypes, such as $\mathrm{Ad} 3, \mathrm{Ad} 4, \mathrm{Ad} 7$, and Ad11, generally cause milder conjunctivitis with systemic involvement. ${ }^{5,6}$ Diagnosis of severe EKC is usually only clinical, but milder forms of the illness due to these other serotypes may require laboratory confirmation. Among those infected with Ad8, eyelid edema may be significantly more common, and serotype Ad8 may persist longer in ocular tissue and secretions than other serotypes. ${ }^{7}$ The fact that Ad8 was found to be the etiologic agent in this outbreak suggests that those patients diagnosed clinically at practice A, without laboratory confirmation, were also likely infected with Ad8.

There is no population based disease surveillance for EKC in Florida or other states, making estimates of disease incidence difficult in the USA. This episode was classified as an outbreak based on the experienced clinician reporting it as such, rather than by comparing disease incidence in practice A to background rates, which are not reliably available for Florida. In Japan, where nationwide sur- veillance has occurred over several years, an estimated 1 million cases of adenoviral conjunctivitis occur each year. ${ }^{5}$ In the USA and elsewhere, outbreaks of EKC are believed to be common, and several outbreak investigations have been described in the literature. ${ }^{1}$ Risk factors for EKC transmission identified in healthcare settings include use of inadequately disinfected tonometers to measure intraocular pressure, ${ }^{8-11}$ use of multi-dose drops,,$^{10}$ or exposure to specific care givers. ${ }^{8-10,12,13}$ Adeno viruses have been shown to persist in the environment and remain viable in a desiccated state for lengthy periods and to be recoverable from the hands of infected individuals, even following hand washing. ${ }^{9,14}$ Guidelines for the disinfection and sterilization of ophthalmology equipment that may contribute to EKC transmission have been published by the Healthcare Infection Control Practices Advisory Committee..$^{15}$

This investigation had several important limitations. Firstly, we only became aware of the outbreak in practice A late in its course, after strict control measures had been implemented. Thus, sampling to identify an environmental source of infection was unlikely to yield useful information and no environmental sampling was conducted. In addition, more than one month had elapsed since physician A became symptomatic, making diagnostic testing of physician A impractical. Only 2 additional cases occurred after the outbreak was reported. Since the outbreak began to come under control at the time the health department investigation was beginning, fewer patients with active symptoms were available for laboratory confirmation to support an epidemiologic investigation of risk factors. Since physician A was the only ophthalmologist in the practice, all patients were exposed to physician A, making measures of association with this exposure difficult to estimate. Therefore, a specific common source of transmission could not be conclusively identified.

When practitioners are confronted with a suspected outbreak of EKC at their facility, there are some recognized best practices, many of which were followed by practice A. They sought laboratory assistance to identify the pathogen, conducted thorough cleaning of the facility and equipment with appropriate disinfectants, segregated symptomatic and non-symptomatic patients, and cooperated fully with the health department in the investigation. These measures appear to have successfully controlled the outbreak. However, healthcare providers with known or suspected EKC should also avoid direct patient contact for 14 days following onset of symptoms in the most recently involved eye,$^{16}$ which practice A did not follow.

Under Florida administrative code 64D-3, any disease outbreak in a community, hospital 
or other institution is reportable to public health authorities. ${ }^{17}$ Our survey of ophthalmologists in the county found that at least 2 other outpatient facilities witnessed a similar number of EKC patients during the same time period, yet neither facility reported these as outbreaks. Without laboratory confirmation and serotyping, it is difficult to determine if the patients diagnosed with EKC seen at these other facilities were somehow linked to patients at practice A. Our findings suggest that passive surveillance based on administrative rule results in poor surveillance sensitivity to detect EKC outbreaks in Florida. The same is likely true in many other states where disease outbreaks are reportable, but individual cases of the same disease are not. Ophthalmologists and other providers experiencing a perceived increase in EKC activity among patients in their facility are encouraged to contact their local health department. Laboratory diagnostic support to confirm certain infectious causes may be available through state health department laboratories.

\section{References}

1. Ford E, Nelson KE, Warren D. Epidemiology of epidemic keratoconjunctivitis. Epidemiol Rev 1987;9:244-61.

2. Herbert L. Conjunctivitis, keratitis and infections of periorbital structures. In: Cohen $\mathrm{J}$ and Powderly WG editors. Infectious Diseases. 2nd edition. New
York: Mosby; 2004:215-223.

3. Rubenstein JB, Virasch V. Conjunctivitis: Infectious and noninfectious. In: Yanoff $M$ and Duker JS editors. Ophthalmology. 3rd edition. New York: Mosby; 2008.

4. Adenovirus. In: Wiedbrauk DL, Johnston S, Johnston LG editors. Manual of Clinical Virology. New York: Raven Press; 1993:54.

5. Aoki K and Tagawa Y. A twenty-one year surveillance of adenoviral conjunctivitis in Sapporo, Japan. Int Ophthalmol Clin 2002;42:49-54.

6. Nicholson F. Introduction to adenoviruses: an overview of morphology, classification and epidemiology. Eye 1993;7:1-4.

7. Roba LA, Kowalski RP, Gordon AT, et al. Adenoviral ocular isolates demonstrate serotype-dependent differences in in vitro infectivity titers and clinical course. Cornea 1995;14:388-393.

8. CDC. Epidemiologic Notes and Reports Epidemic Keratoconjunctivitis in an Ophthalmology Clinic -- California. MMWR Morb Mortal Wkly Rep 1990;39:598-601.

9. Jernigan JA, Lowry BS, Hayden FG, et al. Adenovirus type 8 epidemic keratoconjunctivitis in an eye clinic: risk factors and control. J Infect Dis 1993;167:1307-1313.

10. Viney KA, Kehoe PJ, Doyle B, et al. An outbreak of epidemic keratoconjunctivitis in a regional ophthalmology clinic in New South Wales. Epidemiol Infect 2008;136:1197-1206.

11. Warren D, Nelson KE, Farrar JA, et al. A large outbreak of epidemic keratoconjunctivitis: problems in controlling nosocomial spread. J Infect Dis 1989;160:938-943.

12. D'Angelo LJ, Hierholzer JC, Holman RC, et al. Epidemic keratoconjunctivitis caused by adenovirus type 8 : epidemiologic and laboratory aspects of a large outbreak. Am J Epidemiol 1981;113:44-49.

13. Montessori V, Scharf S, Holland S, et al. Epidemic keratoconjunctivitis outbreak at a tertiary referral eye care clinic. Am J Infect Control 1998;26:399-405.

14. Azar MJ, Dhaliwal DK, Bower KS, et al. Possible consequences of shaking hands with your patients with epidemic keratoconjunctivitis. Am J Ophthalmol 1996;121: 711-712.

15. Rutala WA, Weber DJ and the Healthcare Infection Control Practices Advisory Committee (HICPAC). Guideline for disinfection and sterilization in healthcare facilities 2008. [http://www.cdc.gov/ncidod/dhqp/pdf/guidelines/Disinfection_Nov 2008.pdf]

16. American Academy of Pediatrics: Summaries of infectious diseases. In: editors Pickering LK, Baker CJ, Kimberlin DW, Long SS. Red Book: 2009 Report of the Committee on Infectious Diseases. 28th edition. Elk Grove Village, IL: American Academy of Pediatrics; 2009:206.

17. Florida Department of Health: Administrative rules chapter 64D-3; Control of communicable diseases and conditions which may significantly affect public health. [http:/www.doh.state.fl.us/ disease_ctrl/epi/topics/64D-3_11-08.pdf] 